\title{
Cesium-131 Interstitial Brachytherapy for Recurrent Malignancies of Skull Base
}

\author{
Corey Savard ${ }^{1}$ Gregory Epps ${ }^{2}$ Voichita Bar Ad $^{3}$ Joseph Curry ${ }^{2}$ James J. Evans ${ }^{4}$ \\ Christopher J. Farrell ${ }^{4}$ Gurston Nyquist ${ }^{2}$ Adam Luginbuhl ${ }^{2}$
}

\author{
1 Sidney Kimmel Medical College, Thomas Jefferson University, \\ Philadelphia, Pennsylvania, United States \\ 2 Department of Otolaryngology-Head and Neck Surgery, Thomas \\ Jefferson University, Philadelphia, Pennsylvania, United States \\ 3 Department of Radiation Oncology-Head and Neck Cancer, Thomas \\ Jefferson University, Philadelphia, Pennsylvania, United States \\ ${ }^{4}$ Department of Neurosurgery-Neuro-Oncologic and Stereotactic \\ Surgery, Thomas Jefferson University, Philadelphia, Pennsylvania, \\ United States
}

J Neurol Surg Rep 2019;80:e23-e26.

\author{
Address for correspondence Corey Savard, BS, Sidney Kimmel \\ Medical College, Thomas Jefferson University, 925 Chestnut Street, \\ 6th Floor, Philadelphia, PA 19147, United States \\ (e-mail: Corey.savard@jefferson.edu).
}

\author{
Abstract \\ Keywords \\ - cesium-131 \\ - interstitial \\ - brachytherapy \\ - nasopharyngeal \\ carcinoma \\ - recurrent
}

Background Recurrent malignancy of the skull base poses a treatment challenge due to a lack of treatment options and potential for damage to surrounding structures. Methods Case report of two patients with recurrent nasopharyngeal carcinoma (NPC) of skull base previously treated with adjuvant chemoradiotherapy using intensity-modulated radiation therapy (IMRT).

Results In both cases, the recurrent tumor was treated with endoscopic surgical resection and intraoperative cesium-131 (Cs-131) interstitial brachytherapy (IBT). Total dose delivered to tumor bed was 57 and $60 \mathrm{~Gy}$, respectively. With a half- life of 9 days, the majority of the radiation dose had been delivered within the first 40 days following implant and there have been no treatment-related complications reported.

Conclusion Intraoperative Cs-131 IBT is a feasible adjuvant treatment option for patients with recurrent malignancies of the skull base. These are the first known cases of Cs-131 IBT used for recurrent NPC.

\section{Introduction}

Patients with recurrent malignancy at the skull base pose are a re-treatment challenge. During the course of treatment for skull base malignancies, intensity-modulated radiation therapy (IMRT) provides the backbone of definitive or adjuvant therapy. In the recurrent setting, surgery may be possible. Reirradiation with external beam source remains controversial due to additive toxicity. ${ }^{1}$ Cesium-131 (Cs-131) is a radioactive isotope with favorable dosimetric properties for interstitial brachytherapy (IBT). The mean energy of Cs131 is low and the half-life is 9.7 days, leading to a reduced exposure to the patient, treating providers, and families. ${ }^{2,3}$ The Cs-131 IBT radiation dose is prescribed at a depth of 0.5 to $1 \mathrm{~cm}$; the rapid dose fall limits the dose to surrounding tissues and decreases treatment related side effects. The Cs131 seeds are permanently implanted intraoperatively, eliminating use of catheters required for delivery of high-dose rate (HDR) brachytherapy. Special radiation safety precautions, shielding, or extended hospital care, seen with previous forms of brachytherapy, are generally not necessary ${ }^{2}$. These properties give Cs-131 major benefits over previously used isotopes ${ }^{125}$ I and ${ }^{192}$ Ir in head and neck IBT. In this case series, we present the feasibility, utility, and dosimetry of Cs131 IBT for recurrent skull base tumor in previously irradiated cases. These are the first known cases of Cs-131 IBT use for the treatment of recurrent nasopharyngeal carcinoma (NPC). received

December 20, 2018

accepted

February 20, 2019
DOI https://doi.org/

10.1055/s-0039-1687848. ISSN 2193-6358. (c) 2019 Georg Thieme Verlag KG
Stuttgart · New York

License terms

(1) $\Theta \circledast$ 


\section{Case 1}

A 65-year-old Asian male presented with an erosive mass in the nasopharynx consistent with EBV-encoded small RNA positive (EBER) NPC extending into the cavernous sinus and staged as IIIb. The patient was treated with definitive combined chemoradiation. Definitive IMRT was delivered to the nasopharynx and at-risk cervical lymph nodes to a total dose of $70 \mathrm{~Gy}$, using $2 \mathrm{~Gy}$ daily, 5 days per week concurrently with cisplatin. He also received sequential adjuvant chemotherapy with cisplatinum and 5-flourouracil (5-FU). However, cisplatinum was discontinued due to side effects of persistent neutropenia and bilateral sensorineural hearing loss. In its place, the patient received three cycles of a reduced dose carboplatin and 5-FU.

Three years later, the patient presented with symptoms and imaging concerning for local recurrence of NPC. Biopsy showed fragments of recurrent NPC, nonkeratanizing-undifferentiated type (World Health Organization [WHO] type 3) with strong nuclear staining in-situ hybridization for EBER.
The patient underwent endoscopic nasopharyngectomy with reconstruction with a pediculed nasal septal flap.

Four months after this operation, imaging revealed a recurrent $2.5 \times 2.0 \mathrm{~cm}$ lobulated tissue mass in the skull base. Upon review by the multidisciplinary tumor board, therapeutic treatment was decided to include surgical resection and IBT with Cs-131 intraoperative implants. A preoperative computed tomography (CT) scan (-Fig. 1A) was used to plan the placement of the cesium seeds and expected dose.

Primary surgical intervention consisted of a resection of the lateral sphenoid, posterior maxilla extending to the foramen rotundum superiorly and resection of the pterygoid plates inferiorly. Reconstruction and Cs-131 strand implantation was staged and undertaken the following day utilizing a left temperoparietal fascia flap, and infratemporal fossa approach to left nasopharyngeal tumor resection defect with endoscopic placement of brachytherapy to the at-risk surgical bed. Coverage and stabilization of the Cs-131 strand was achieved utilizing the left temporoparietal fascia flap.

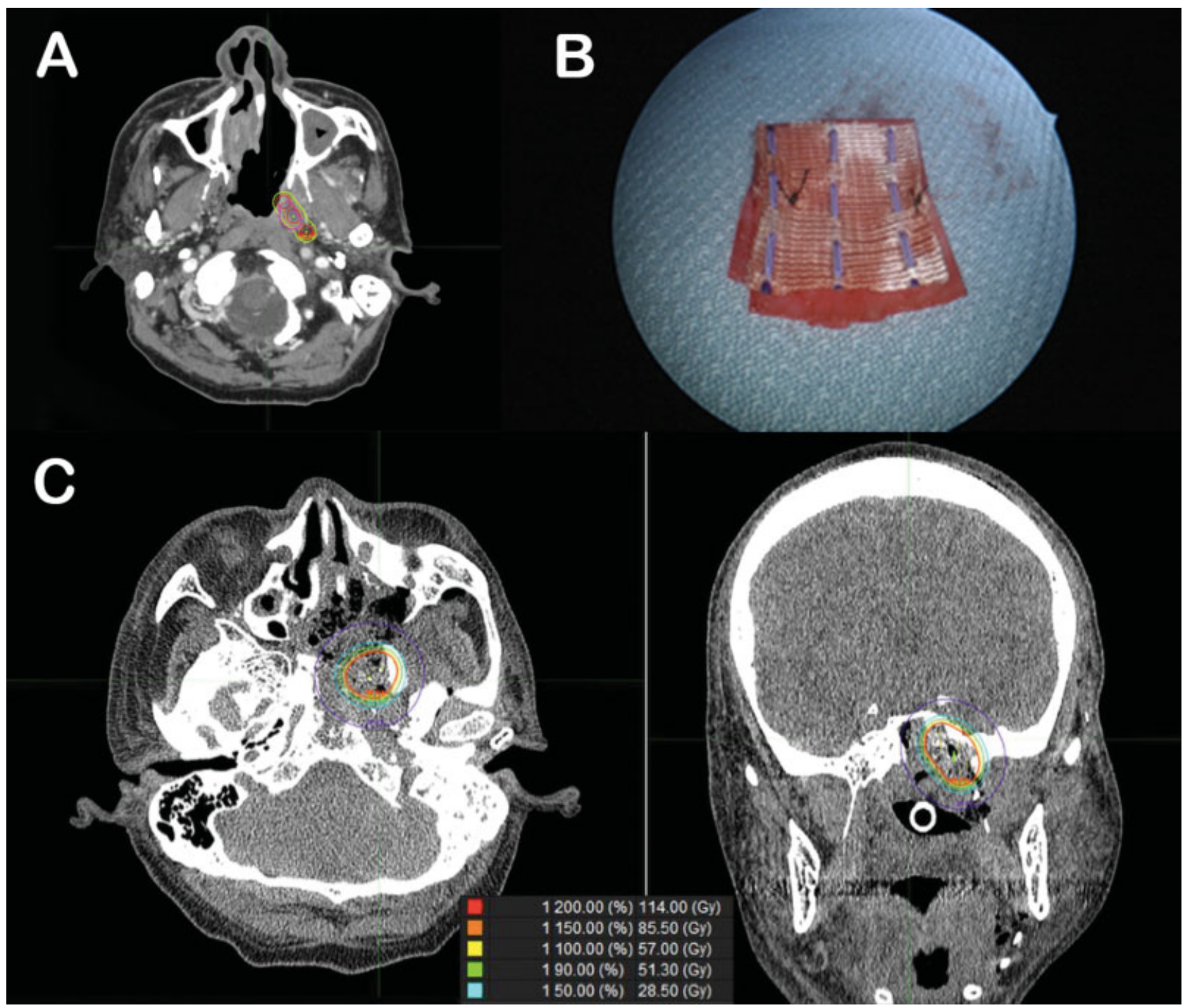

Fig. 1 Case 1 (A) Preoperative dose planning axial CT. (B) Vicryl mesh with suture containing cesium brachytherapy seeds used in both cases 1 and 2. (C) Postoperative CT scans with dosimetry curves. CT, computed tomography. 
Brachytherapy preimplant plan was performed by the radiation oncology team using a Vicryl mesh (-Fig. 1B), which contained brachytherapy seeds, two strands of three seeds, for a total of six Cs-131 seeds. The mesh was sutured endoscopically to the skull base and nasopharynx followed by its coverage using a tunneled temporoparietal fascia flap. Pathology confirmed recurrent CK5/6+ and EBER+ NPC involving dense connective tissue and bone in left nasopharynx and skull base. The radiation dose prescribed consisted of a total dose of $57 \mathrm{~Gy}$ at $5 \mathrm{~mm}$ depth with majority of the dose delivered over the first 40 days. Six seeds of Cs-131 with an activity of $2.7 \mathrm{mCi}$ were used for IBT for this case.

Post-operative CT scans performed within 24 hours postimplant (-Fig. 1C) showed placement of the seeds in the resection bed and verified doses of radiotherapy.

He is currently 9 months out from surgery with no signs of recurrent disease. His post treatment positron emission tomography (PET) scan and MRI demonstrate no evidence of metabolically active metastasis or recurrent disease. He will be closely followed-up in the coming months.

\section{Case 2}

A 34-year-old man presenting with right sided nasal obstruction and epistaxis was found to have a large nasopharyngeal mass with biopsy-proven EBER+, nonkeratanizing undifferentiated NPC with extension into right pterygopalatine fossa, stage III. He underwent a PET-CT which showed no distant metastasis and started on definitive combined chemoradiation. Definitive IMRT consisted of a total dose of $70 \mathrm{~Gy}$, using a 2 Gy daily, 5 days per week regiment to the nasopharynx and at-risk cervical lymph nodes. He also received sequential adjuvant chemotherapy with cisplatinum at $100 \mathrm{mg} / \mathrm{m}^{2}$ dose over 21 days.

Following the completion of his adjuvant chemoradiation, he underwent surveillance MRI and PET-CT at 8 and 12 weeks post treatment which were negative for local recurrence, metabolically active malignancy, or distant metastatic disease. Despite negative imaging results, there was persistent swelling in the right superior nasopharynx 4 months following completion of adjuvant therapy. This area was biopsied and found to be persistent EBER positive, nonkeratanizing undifferentiated NPC. Upon review in the multidisciplinary clinic, therapeutic treatment was decided

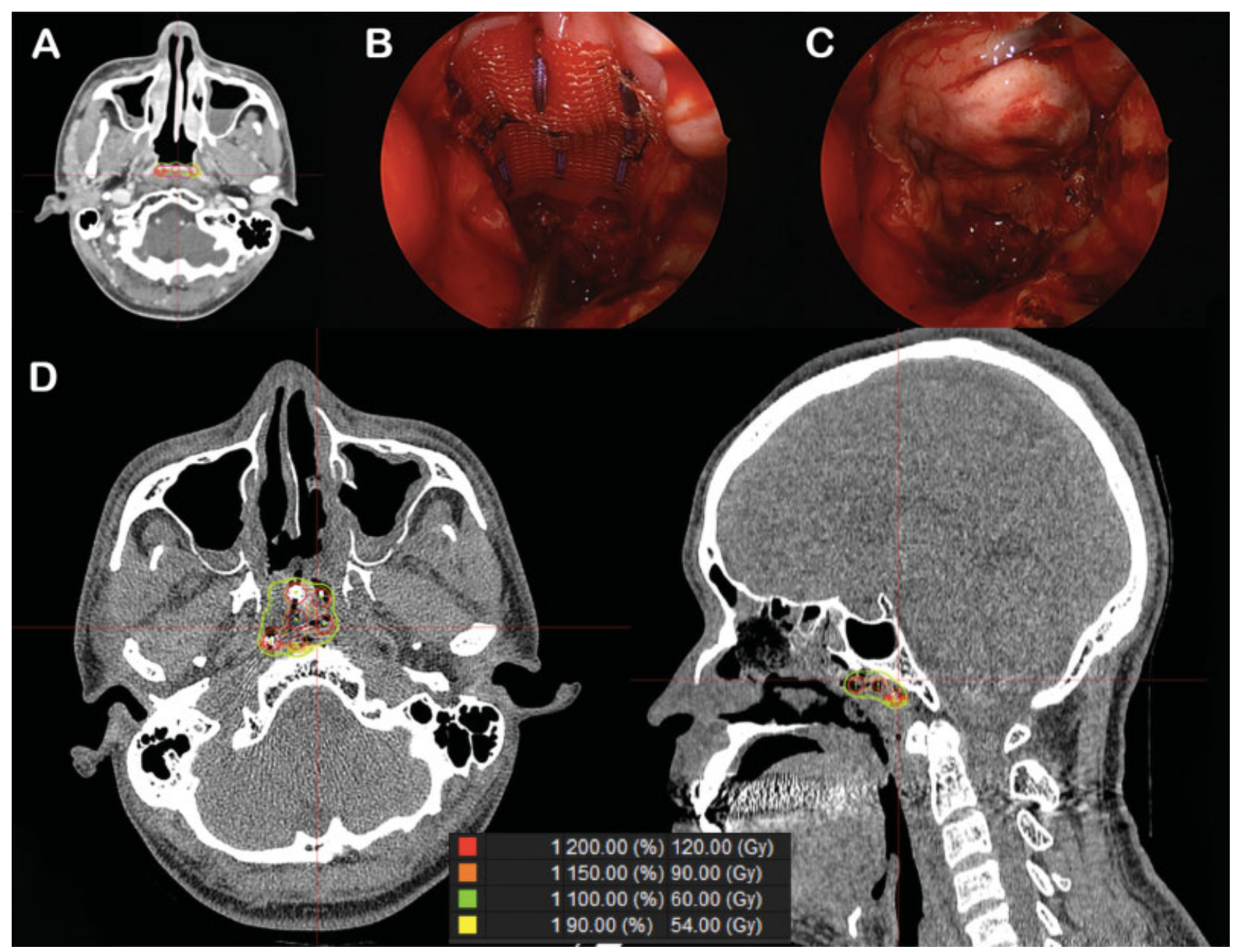

Fig. 2 Case 2 (A) Preoperative dose planning axial CT, (B) intraoperative endoscopic image of mesh and synthetic dura, (C) intraoperative endoscopic image of nasal septal flap covering mesh, (D) postoperative CT scans with dosimetry curves. CT, computed tomography. 
to proceed with surgical resection and IBT with Cs-131 intraoperative implants. A preoperative CT ( $\mathbf{- F i g . ~ 2 A ) ~ s c a n ~}$ was used for the Cs-131 preimplant plan.

Surgical resection involved an endoscopic transnasal approach to the cranial base with posterior septectomy and resection of neoplastic tissue of nasopharynx along clivus and skull base. Following confirmation of negative margins, reconstruction was planned to incorporate placement of Cs-131 seeds and a left sided vascularized pediculed nasal septal flap. Cs-131 seeds were prepared by radiation oncology team using a Vicryl mesh (-Fig. 1B). It contained three separate strands of three seeds each, for a total of nine Cs-131 seeds. Following resection, bony skull base remained exposed. To separate the mesh from the bony skull base and reduce the risk of osteoradionecrosis, a folded piece of $1 \times 3$ inch Dura Repair was sutured to the mesh.

The mesh and synthetic dura (-Fig. 2B) were endoscopically placed, covering the resection bed. The nasal septal flap ( $\mathbf{F i g . ~ 2 C ) ~ w a s ~ r o t a t e d ~ t o ~ c o v e r ~ t h e ~ b o n y ~ c r a n i a l ~ b a s e ~ a l o n g ~}$ the clivus and middle cranial fossa and to entirely cover the cesium seeds. Final pathology confirmed persistent EBER positive, nonkeratanizing undifferentiated NPC resected to negative margins.

The prescribed radiation dose was $60 \mathrm{~Gy}$ at $5 \mathrm{~mm}$ depth with the majority being delivered over the first 40 days. Nine seeds of Cs-131 with an activity of $2.7 \mathrm{mCi}$ were used for this IBT. Postoperative CT scans (-Fig. 2D) showed placement of the seeds in the resection bed and the post implant radiotherapy plan.

The patient is currently being followed with serial imaging and physical exams without evidence of recurrence.

\section{Discussion}

Tumors of the skull base are particularly challenging for clinicians. The density of high-risk structures at the skull base and incredible topography for tumor spread leads to incomplete resection and a poor prognosis. Due to previous treatments including chemoradiotherapy, the therapeutic options available are often limited. Reirradiation has the potential to cause cerebrovascular accidents, carotid rupture, skin necrosis, and spinal cord damage. Chemotherapy given as single-modality treatment for these patients has a limited benefit ${ }^{1}$. The theoretical benefit of IBT, as an adjunct to total resection, is improved control of malignancy via targeted delivery of irradiation to the tumor bed. The dosimetric properties of Cs-131 previously outlined make it ideally suited for IBT at the skull base, significantly reducing the radiation dose to the surrounding critical normal structures, and the risk of radiation-related side effects.
Considering the skull base has limited soft tissue and difficult access, stabilization of the cesium seeds poses a significant challenge. Migration of the radioisotope seeds may reduce the effectiveness of the therapy and increase the dose to normal structures causing unintended toxicities. Migration of the seeds into the aerodigestive tract could result in additional procedures necessary to retrieve the seeds or may cause damage to the mucosa. The use of mesh containing the Cs-131 seeds and adequate surgical techniques to stabilize the implant may eliminate the risks of these severe complications.

Reconstruction needs to be considered to support the seeds if placed in a nondependent position. Soft tissue membranes may also be needed to protect critical structures, such as the carotid, cranial nerves, or bones from direct contact with the Cs-131 seeds. Given these concerns, a synthetic dura was used as a protective buffer in case 2 . Careful consideration was taken to ensure the dose dependent distance of the Cs-131 seeds to the at-risk surgical bed was optimized for treatment effect while limiting the risk of osteoradionecrosis.

\section{Conclusion}

In conclusion, this case report highlights the first ever use of a novel approach to targeted intraoperative radiation therapy by using Cs-131 permanent interstitial implant in the previously radiated recurrent malignancies at the skull base.

\section{Financial Disclosure}

The authors have indicated they have no financial relationships relevant to this article to disclose.

Conflict of Interest

None declared.

\section{References}

1 Kupferman ME, Morrison WH, Santillan AA, et al. The role of interstitial brachytherapy with salvage surgery for the management of recurrent head and neck cancers. Cancer 2007;109(10): 2052-2057

2 Parashar B, Wernicke AG, Pavese A, et al. Cesium-131 permanent seed brachytherapy: dosimetric evaluation and radiation exposure to surgeons, radiation oncologists, and staff. Brachytherapy 2011;10(06):508-513

3 Armpilia CI, Dale RG, Coles IP, Jones B, Antipas V. The determination of radiobiologically optimized half-lives for radionuclides used in permanent brachytherapy implants. Int J Radiat Oncol Biol Phys 2003;55(02):378-385 